CONTEXTO

ISSNPrint: 2339-3084

ISSN (E): 2346-0784

http://contexto.ugca.edu.co

Investigación

Información del artículo

Recibido: 20/10/2019

Revisado: 29/11/2019

Aceptado: 10/12/2019

Información del autor

* Universidad La Gran Colombia, programa de Relaciones Internacionales y Comercio Justo

Correspondencia

Norfaliaalexandra.benitez@ ulagrancolombia.edu.co

(C) 2019 Universidad La Gran Colombia. Este es un artículo de acceso abierto, distribuido bajo los términos de la licencia Creative Commons Attribution License 4.0, que permite el uso ilimitado, distribución y reproducción en cualquier medio, siempre que el autor original y la fuente se acrediten.

Cómo citar

Benitez, N.A., \& Ciro, S. (2019) Análisis del mercado Colombo-Méxicano del clúster de cosméticos de Bogotá. Contexto 8, 4-17.

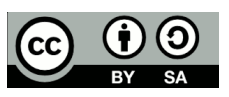

\section{Análisis del mercado Colombo-Mexicano del clúster de cosméticos de Bogotá}

\author{
Norfalia A. Benitez* , Santiago Ciro*
}

\section{Resumen}

El sector de cosméticos en Colombia ha venido en crecimiento los últimos años (2009-2018) y ha tomado un gran reconocimiento en el mercado internacional, el clúster de cosméticos de Bogotá ha sido creado para apoyar a las empresas del sector, incentivarlas y crear una agremiación que comparta una misma visión del futuro y se apoye entre sí. El presente trabajo muestra la dinámica del mercado Colombo-mexicano para la pequeña y mediana Empresa (Pymes) del clúster de cosméticos en Bogotá, se plasman las ventajas y desventajas, así como el comportamiento de las Pymes y las características del mercado del clúster de cosméticos de Bogotá.

Palabras clave: Cosméticos, clúster, mercado, comercio internacional.

\section{Analysis of the Colombo-Mexican Market of the Cosmetics Cluster of Bogotá}

\begin{abstract}
The sector of cosmetics in Colombia has been growing the past few years and has taken a great recognition in the international market, Bogotá cosmetics cluster has been created to support the companies in the sector, encourage them and create an association that share the same vision of the future and support each other. The present work shows the dynamics of the market Colombo-Mexican for the small and medium-sized enterprises (SMEs) in the cluster of cosmetics in Bogota, are reflected the advantages and disadvantages, as well as the behaviour of SMEs and the characteristics of the market of the cluster of cosmetics in Bogotá.
\end{abstract}

Keywords: Cosmetics, Cluster, market, International Trade. 


\section{Introducción}

El proceso de globalización trae consigo una articulación de los mercados a través de acuerdos comerciales o tratados de libre comercio que a su vez generan grandes oportunidades y retos para las economías nacionales, así mismo se hace necesario convertir esta aparente amenaza para los sectores más sensibles de nuestro país en una ineludible oportunidad desde el ejercicio académico. El sector cosmético del país ha venido tomando relevancia en los últimos años(2009-2018), el país apuesta para fortalecer la economía de las regiones e impulsar el comercio internacional de este sector, aprovechando la actual ola de interés por consumo en productos cosméticos hechos con bases naturales y los diferentes tratados de libre comercio vigentes que facilitan el intercambio comercial.

Colombia ha mantenido una relación comercial con México por más de una década y la balanza comercial ha sido deficitaria para Colombia, con el ánimo de fortalecer la relación comercial y teniendo en cuenta las características del mercado mexicano, las empresas Cosméticas del Clúster han empezado a ver a México como un país para incursionar con sus productos.

México es el décimo país más poblado del mundo con 129’163.000 habitantes al año 2017 según informó la ONU. (Excelsior, 2017) A la vez el mismo año un estudio de consumo y uso de cosmético mostró que el 79,2 \% de las personas de México utiliza algún producto para el cuidado de la piel. (Canipec, 2017) Lo que permite suponer que hay un gran mercado de consumidores para productos cosméticos, además Procolombia publicó que dentro de las manufacturas con oportunidades de negocio para México se encuentra los cosméticos junto con dotación hotelera, Kitchenware y dinnerware, Muebles RTA y de alta gama, resaltando que los productos genéricos con precios al alcance del bolsillo de los hogares son preferidos a la hora de comprar. (2015)

En 1992 empresarios de Colombia y México se unieron para crear lo que se conoce como cámara de comercio e integración Colombo Mexicana, esta entidad ofrece asesorías en aspectos comerciales, solución a consultas sobre el TLC del G3 y Alianza del Pacífico, colaboración con trámites de visas entre otros servicios que facilitan el comercio entre los dos países.

En el año 2009 el sector cosmético se incluyó en el Programa de Transformación Productiva (PTP) del Ministerio de Comercio Industria y Turismo, con el propósito de dar más apoyo al sector para que sea más competitivo en los mercados globales, aprovechando que Colombia es el segundo país con más biodiversidad del mundo (Propais, 2013) en concordancia con esto y con el propósito de fortalecer y desarrollar una economía sostenible regional en el país, se han creado varios clúster que apoyan a diferentes gremios, estos clúster permiten el diseño y ejecución de proyectos asociativos que generan mayor confianza, redes de trabajo y capital social, lo que contribuye a la apuesta del país por un desarrollo regional. (Innpulsa, Gobierno de Colombia, ClusterDevelopment, \& UniversidaddelRosario, 2018). Un dato para resaltar es que "Colombia se ubica como la cuarta economía y el cuarto mercado más grande en América Latina en productos de belleza y cuidado personal. En su orden, los primeros son Brasil, México y Argentina." (Camara de comercio de Bogotá, 2018) Se evidencia que la posición geoestratégica con la que cuenta Colombia le supone una ventaja predominante frente a 
otros mercados de la región, de igual manera su posición geográfica proporciona una ventaja de fauna y flora con la que acentúa un gigantesco potencial para producir excelentes productos cosméticos y de aseo, aprovechando y resaltando los beneficios de productos naturales únicos que destacan por calidad, beneficios y exclusividad.

La investigación gira en torno al análisis del mercado colombo-mexicano del clúster de cosméticos de Bogotá; se aplicó una entrevista a profundidad ${ }^{1}$ con preguntas abiertas lo cual nos permitió identificar las ventajas y desventajas del sector frente a las oportunidades de negocio con los vigentes acuerdos comerciales con México. Aunque en México las marcas más consumidas de cosméticos pertenecen a grandes empresas multinacionales, se encuentran dos tendencias que sirven de estrategias para las empresas del clúster de cosméticos de Bogotá por un lado está la tendencia del consumo natural en cosméticos ( productos libres sosa, parabenos, petrolatos y maltrato animal) (El Economista, 2017) y por otro los productos genéricos (productos que pueden ser usados por todos los miembros de la familia)con precios asequibles apetecidos por el mercado.

Teniendo en cuenta lo anterior es pertinente preguntarse ¿Cómo es la dinámica del mercado Colombo-mexicano para la pequeña y mediana Empresa (Pymes) del clúster de cosméticos en Bogotá?

\section{Antecedentes}

El clúster de cosméticos ha sido una iniciativa para fortalecer la competitividad del sector cosméticos de la región articulando a las empresas, academia y gobierno con el fin de potencializar esta industria y abastecer las necesidades a nivel nacional y en América Latina, además como lo menciona la (Cámara de Comercio de Bogotá) el sector se encuentra "en línea con los esfuerzos de Política Industrial Moderna que viene desarrollando el país en el marco del Sistema Nacional de Competitividad, Ciencia, Tecnología e Innovación". El sector cosmético a nivel global se está enfocando en productos hechos con bases naturales y Colombia es un país con gran diversidad de materia prima natural para la fabricación de estos productos que tienen gran demanda. En 2017 pasado el sector cosmético y de aseo tuvo un incremento de 8,38\% frente a 2016 y cerró el mercado con US\$3.391 millones en el subsector de cosméticos (Portafolio, 2018).

Los tratados de libre comercio brindan oportunidades para países en desarrollo como Colombia, además, "es un lugar común comprender a la globalización como un proceso de interconexión de las economías y las empresas, así como una creciente conectividad en la tecnología y el conocimiento (Batres \& Calderon, 2007). Como menciona Krugman "Si consideramos que el mercado es algo bueno, en ese caso el poder de la globalización para producir riqueza y, especialmente, para elevar e incorporar con rapidez a países pobres al mundo moderno nos mueve y nos ínsita a alabarla" (2007).

1. Encuentros cara a cara entre el investigador y los informantes, encuentros dirigidos hacia la comprensión de las perspectivas que tienen los informantes respectos de sus vidas, experiencias o situaciones, tal como las expresan con sus propias palabras. Las entrevistas en profundidad siguen el modelo de una conversación entre iguales, y no de un intercambio formal de preguntas y respuestas. (Introducción a los métodos cualitativos en investigación. Taylor,S.J. Bogdan, R) 
En relación con los clúster encontramos que estos son un eje fundamental y estratégico para el crecimiento económico de las empresas y de las regiones que los implementan:

Los departamentos que han direccionado sus cadenas productivas a la conformación de clústeres han mejorado sus niveles de competitividad y productividad, lo cual ha incrementado su participación en el PIB nacional, que en su mayoría se debe a la organización de su producción y comercialización a través de los diferentes clúster que se han integrado en las regiones, el territorio Departamento que más le aporta al PIB nacional sigue siendo la ciudad de Bogotá con un 26,1\%, esto se debe a la labor que está realizando la Cámara de Comercio apoyando a los diferentes clústeres que se organizaron en la ciudad. (Arana \& Ballesteros, 2016).

\section{Relación comercial Colombia-México}

Actualmente (2018) Colombia mantiene un TLC con México, puesto en vigencia desde Enero de 1995 y también mediante la Alianza del pacifico creada en el año 2011 y donde participan también Chile y Perú, estos acuerdos dan facilidad para el intercambio de bienes y servicios con este país, sin embargo Colombia ha mantenido una balanza comercial deficitaria frente a México, circunstancia que ha permitido al sector de cosméticos empezar a contemplar este país para sus exportaciones "las industrias empezaron a profundizar sus negocios en otros países, especialmente los de la Alianza del Pacífico. De esta manera, se han incrementado exportaciones a México y Chile." (Portafolio, 2018).

“En el año 2016 Colombia exportó US\$630 millones con un descenso del 17\% frente a 2015" (Dinero, 2017)sin embargo se tuvo una recuperacion en el año sigueinte; "Finalmente, lograron crecer más del 15\%, al cierre del 2017". (Portafolio, 2018)

Tabla 1. Balanza comercial con Alianza del Pacífico (miles UD\$ FOB)

\begin{tabular}{lrrrr}
\hline & Año completo & \multicolumn{3}{c}{ enero-mayo } \\
\cline { 2 - 5 } Países & 2016 & 2017 & 2017 & 2018 \\
Chile. & -14962 & 373921 & 138754 & 192883 \\
Perú. & 392180 & 496597 & 157216 & 163859 \\
México. & -2361256 & -1788496 & -912084 & -823057 \\
Total General & -1984038 & -917978 & -616115 & -466315 \\
\hline
\end{tabular}

Nota. Recuperado de "Estadísticas de comercio exterior", de Mincit., (2018). Recuperado de http://www.mincit.gov.co

En la tabla 1 se evidencia el histórico de la balanza comercial Colombiana frente a los países que integran la alianza del pacífico, por tanto en el año 2016 se nota que la balanza comercial es deficitaria 
frente a Chile y México, sin embargo para los años 2016-2017 y hasta mayo de 2018 ha venido consolidando un superávit en la balanza comercial con Perú; México supone una ventaja competitiva en cuanto a exportaciones frente a Colombia si bien la balanza comercial ha venido en los últimos dos años disminuyendo gradualmente el déficit es evidente que esta situación en la relación comercial se mantiene.

Tabla 2. Balanza Comercial por Países- Colombia (Enero-Diciembre, Millones US\$)

Nota. Recuperado de "Informe", de ANDI.,(2018). Recuperado de http://www.andi.com.co

En la tabla 2 observa que la balanza comercial con México para el año 2017 disminuyó en 573 Millones de US\$, y con base en los datos registrados con Chile y Perú se logró un superávit comercial al cierre del año 2017.

\section{Clústeres}

Según Porter los Clúster "son agrupaciones de empresas, instituciones y negocios que se dan en concentraciones geográficas y que están interconectadas en una actividad específica: estas incluyen los proveedores, las empresas, los distribuidores, las cuales realizan sus actividades de apoyo y primarias en función del cliente aumentando valor para sus empresas." (1998). 
Otros autores mencionan algunas de las ventajas que proporcionan los clústeres:

Los clúster han sido reconocidos como escenarios en los que se dan condiciones muy favorables ya sea para el estímulo de la productividad y de la innovación en las empresas que los integran, ya sea para la formación de los recursos humanos o para la creación de nuevos negocios (Capó, Expósito, \& Tomás, 2007).

De acuerdo con Red Clúster Colombia en (2013) mediante la iniciativa de origen privado se creó el clúster de cosméticos de Bogotá, liderado por la Cámara de comercio de Bogotá y cuenta con la participación de otras entidades entre ellas el SENA, ANDI, Universidad Nacional de Colombia, Procolombia entre otras.

\section{Clúster de cosméticos de Bogotá}

La cámara de comercio de Bogotá en los últimos años ha impulsado la iniciativa de creación de clúster en la ciudad con el fin de fortalecer el tejido empresarial de los diferentes actores económicos.

Colombia es uno de los principales proveedores de cosméticos y productos de aseo de la región, la industria ha mostrado un crecimiento sostenible los últimos 8 años en cuanto a producción, ventas y exportaciones. Las ventas de maquillaje, tratamientos para la piel, cremas, jabones, shampoo colombianos gozan de gran prestigio en el exterior por su calidad y sus ingredientes naturales. (Procolombia, s.f)

En la Figura 1 (más adelante) se muestra un esquema de algunos datos relevantes del clúster, que ilustran en retrospectiva lo que ha significado este clúster para el empresariado del cluster al año 2018.

Figura 1. Ficha Técnica del Clúster de Cosméticos de Bogotá

Ficha Técnica

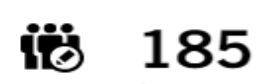

Actores participantes

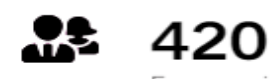

Empresarios beneficiados en

proyectos del Cluster

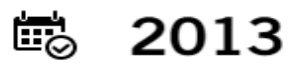

Año de creación de la Iniciativa de Cluster

\section{of $3.3 \%$}

Aporta la industria cosmética al PIB

Nota. Recuperado de "ficha técnica", de CCB (2018). Recuperado de https://www.ccb.org.co 
Tabla 3. Método

\begin{tabular}{ll}
\hline \multicolumn{1}{c}{ Tipo de Investigación } & \multicolumn{1}{c}{ Descriptivo } \\
\hline Método-Enfoque & Cualitativo \\
$\begin{array}{ll}\text { Fuentes de Recolección e } \\
\text { Información }\end{array}$ & $\begin{array}{l}\text { Primaria: Entrevistas a } \\
\text { empresas } \\
\text { Secundaria: Páginas Web, } \\
\text { Catálogos Digitales. }\end{array}$ \\
Método de recolección & Entrevista a profundidad \\
Población & $\begin{array}{l}\text { Empresas Cluster de } \\
\text { Cosméticos de Bogotá }\end{array}$ \\
Muestra & 2 \\
Duración entrevistas & 45 minutos \\
\hline
\end{tabular}

Nota. Elaboración propia

La investigación descriptiva "comprende la descripción, registro, análisis e interpretación de la naturaleza actual, y la composición o proceso de los fenómenos. El enfoque se hace sobre conclusiones dominantes o sobre grupo de personas, grupo o cosas, se conduce o funciona en presente". (Tamayo \& Tamayo, 2002)

Se escogió la entrevista en profundidad por su flexibilidad para la recolección de información y teniendo en cuenta que es acorde a esta investigación de enfoque cualitativo.

“Como la observación participante, las entrevistas cualitativas requieren un diseño flexible de la investigación. Ni el número ni el tipo de informantes se especifica de antemano. El investigador comienza con una idea general sobre las personas a las que entrevistará y el modo de encontrarlas, pero está dispuesto a cambiar de curso después de las entrevistas iniciales. Es difícil determinar a cuántas personas se debe entrevistar en un estudio cualitativo. Algunos investigadores tratan de entrevistar al mayor número posible de personas familiarizadas con un tema o acontecimiento." (Taylor \& Bogdan, 1992)

\section{Mercado cosmético colombiano}

El mercado cosmético de Colombia ha tomado una gran relevancia en los últimos años, así como un buen reconocimiento de productos cosméticos especialmente en el mercado internacional. En 
la tabla 3(más adelante) se puede observar que, frente a la relación comercial del sector cosmético de México para Colombia, México es el principal proveedor de productos cosméticos, México es el tercer país que exporta Colombia dichos productos, antecedido por Perú y Ecuador respectivamente.

Tabla 4. Principales Destinos de Exportación y de Proveedores para Colombia

\begin{tabular}{ccc}
\hline \multicolumn{3}{c}{ Años 2013-2017 } \\
\hline Puesto & Exportación & Importación \\
1 & Perú & México \\
2 & Ecuador & Estados Unidos \\
3 & México & Brasil \\
4 & Chile & España \\
5 & Panamá & Francia \\
6 & República & República Dominicana \\
7 & Dominicana & Guatemala \\
8 & Costa Rica & Uruguay \\
9 & Bolivia & Suiza \\
10 & Guatemala & Argentina \\
\hline
\end{tabular}

Nota. Elaboración propia, datos tomados de Trademap.

Respecto al consumo interno de productos cometidos se evidencia que los consumidores colombianos prefieren comprar productos de marcas extranjeras al asociarlos con productos de mejor calidad por la investigación y desarrollo; en un estudio realizado por Nielsen se determinó que:

Cuatro de cada 10 consumidores prefieren el shampoo y acondicionador (44\%), los desodorantes (44\%), los cosméticos (40\%), de marcas globales. En cuanto a cremas para manos y cuerpo, y jabón, la preferencia es similar para 3 de cada 10 encuestados. En algunas categorías de Aseo Personal las marcas globales, por su escala, tienen altas inversiones en Investigación y Desarrollo, lo que les da ventajas en cuanto a calidad, variedad e innovación. (Nielsen, 2016).

En otro estudio realizado por Linio para conocer los países donde los cosméticos (maquillaje, cremas, tintes, perfumes y otros) son más económicos se halló que "En América Latina, Colombia es el país donde estos servicios son más baratos, seguido de Panamá y México". (CNN Español, 2017)

A continuación se muestran algunos de los productos cosméticos más consumidos por las colombianas: 
Tabla 5. Productos cosméticos de maquillaje más comprados en Colombia

\begin{tabular}{l}
\hline \multicolumn{1}{c}{2012} \\
\hline Pestañina \\
Polvos Compactos \\
Labiales \\
Gloss \\
Esmaltes \\
\hline
\end{tabular}

Elaboración propia datos tomados de La Republica sobre un estudio de natura con Ipsos-Napoleón Franco.

\section{Mercado cosmético mexicano}

México es un país con un gran mercado de consumidores de productos cosméticos no solo por la densidad poblacional sino también un por un factor cultural en donde la apariencia física tiene gran importancia, tanto así que:

México se ubica en el puesto 14 de países importadores del mundo en productos de cosméticos y aseo y en el puesto 1 a nivel de América Latina. En México los consumidores prefieren comprar en grandes almacenes que, a través de la venta directa, e-commerce y homeshopping, también prefieren productos hechos con ingredientes naturales y con nuevas fórmulas mejoradas que indiquen calidad. (Procolombia, 2018).

Según estudio realizado por Nielsen en México más del 50\% de los encuestados reconocen y perciben que las marcas globales son mejores en cuanto a tecnología e innovación comparadas con las marcas locales. "En el caso de productos de uso personal y para el hogar, $51 \%$ de los consumidores prefiere comprar shampoo para el cabello, $45 \%$ jabones, $46 \%$ cremas y lociones para el cuerpo, $50 \%$ cosméticos y $51 \%$ desodorantes de marcas extranjeras". (2016).

Actualmente los mercados emergentes como México vienen afianzando sus relaciones comerciales frente al mundo en donde prima el intercambio equivalente, pero de recíproco beneficio, en consecuencia, es evidente que las relaciones comerciales se van incrementando por una parte por la cercanía, y por otra por acuerdos comerciales preestablecidos. Actualmente, Colombia es el tercer país destino de las exportaciones de cosméticos mexicanos pero en cuanto a la importación de estos, Colombia ocupa el puesto número 7 dentro de sus principales proveedores, como lo muestra la Tabla 5 (más adelante). 
Tabla 6. Principales Destinos de Exportación y de Proveedores para México.

\begin{tabular}{lll}
\hline & \multicolumn{2}{l}{ Años 2013-2017 } \\
\hline Puesto & Exportación & Importación \\
1 & Estados Unidos & Irlanda \\
2 & Guatemala & Estados Unidos \\
3 & Colombia & Francia \\
4 & Argentina & España \\
5 & Chile & Uruguay \\
6 & Perú & China \\
7 & Brasil & Colombia \\
8 & Costa Rica & Italia \\
9 & Canadá & Reino Unido \\
10 & Alemania & Brasil \\
\hline
\end{tabular}

Elaboración propia, datos tomados de Trademap.

Si bien el tejido empresarial mexicano está compuesto en su mayoría por empresas extranjeras hoy es evidente que ese papel de país maquilador de productos cosméticos lo logran posicionar como uno de los países más promisorios de la región Latam (Latinoamérica), en consecuencia según datos publicados por Canipec "Las empresas de cuidado personal afiliadas a Canipec, durante 2016 exportaron: 2,691.6 millones de dólares, importaron 1,524.6 millones de dólares y reportaron un superávit de 1,167.0 millones de dólares". (2017), además "México ocupa el tercer lugar en producción de cosméticos, superado únicamente por Brasil y Estados Unidos, segundo y primer puesto, respectivamente". (Expo Belleza Fest., 2018).

En cuanto al consumo de productos cosméticos de maquillaje los de mayor demanda son los de la figura 2:

Figura 2. Productos cosmeticos de maquillaje mas comprados en México

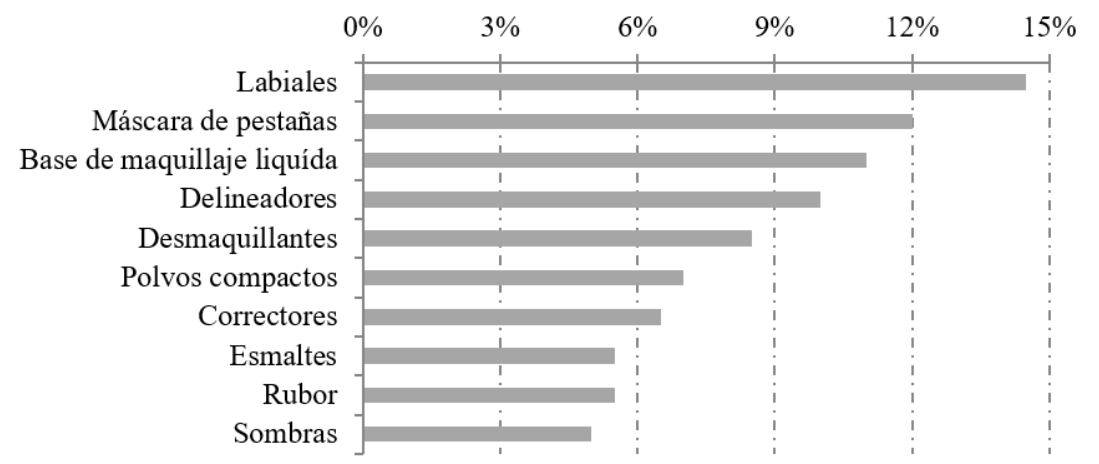

Nota. Recuperado de “¿Cuáles son los cosméticos que más se compran en México?”, de Merca2.0., (2017). Recuperado de https://www.merca20.com 


\section{Resultados}

En esta sección presentamos los hallazgos encontrados como resultado de la investigación principalmente de fuentes secundarias y de las entrevistas aplicadas.

-No hay estudios previos que permitan configurar un análisis más detallado para el mercado cosmético de Colombia, en contraste a la diversa y completa información pública del sector cosmético mexicano, además la poca información que se encuentra es de más de cuatro años atrás, convirtiéndose en información poco confiable.

- Los consumidores actualmente prefieren productos hechos a base de productos naturales y aquellos que perciben con mayor calidad.

- Al momento de comprar un producto cosmético tanto colombianos como mexicanos prefieren aquellos de marcas extranjeras, pues los catalogan como mejores por la investigación y desarrollo que tienen o pueden tener.

- La posición geoestratégica con la que cuenta Colombia permite suponer una ventaja predominante frente a otros mercados de la región, de igual manera su posición geográfica proporciona una ventaja de fauna y flora con la que acentúa un gigantesco potencial para producir excelentes productos cosméticos y de aseo.

- Los entrevistados consideran que uno de los hechos que más los han afectado fue la carga tributaria impuesta a los productos de valor agregado pasando de un IVA del 16\% al 19\%, lo que influye en la dinámica de su rotación y compra, en palabras de Sandra Pascuas, Jefe Comercial y operativa de Callegari plásticos "Desde que se aumentó el IVA al 19\%, se ha bajado un poco el mercado de los empaques en general" y de María del pilar Rojas de Del Mar Laboratorios "Afectó muchísimo el 19\% que le subió el gobierno al impuesto, demasiado. Esta es la hora que todavía no nos hemos podido levantar."

- El conglomerado de empresas que constituyen lo que se conoce como Clúster de cosméticos de Bogotá hoy reconocen que por tratarse de un sector en su mayoría compuesto por micro y pequeñas empresas su capital para inversión en desarrollo e investigación para nuevos productos se ve disipada.

- Los entrevistados por la experiencia y conocimiento del sector y del mercado, afirman que los colombianos prefieren comprar productos extranjeros ya que los perciben de mayor calidad y confianza a la hora de la compra.

\section{Conclusiones}

Colombia tiene un gran mercado para el consumo de productos cosméticos y una cultura de vanidad no solo en mujeres sino también en hombres, además se encuentra una gran cantidad de empresas cosméticas que trabajan por posicionarse en el mercado local y buscan nuevas oportunidades de 
expandirse en el mercado internacional especialmente en Latino América; sin embargo, uno de los principales factores para el éxito de la comercialización de productos son los precios para el consumidor final, estos deben ser asequibles y tener una buena relación calidad/precio. Los consumidores actualmente buscan estar a la vanguardia de las tendencias en belleza y valoran la innovación en los productos.

Actualmente el sector cosmético cuenta con un gran potencial en el desarrollo de productos elaborados con bases naturales, no solo en el caso del clúster de Bogotá sino de todo el sector a nivel nacional, teniendo en cuenta que uno de los retos más grandes está en cómo cautivar e incentivar la demanda del producto hecho en Colombia y disminuir la percepción de que los productos extranjeros son de mejor calidad. Las empresas nacionales en su gran mayoría no cuentan con disposiciones presupuestales para invertir en investigación y desarrollo, sin embargo, se interesan por ofertar productos de excelente calidad, de hecho en situaciones de dificultades económicas lo último que se sacrifica es la fórmula del producto, dejando como primera opción la reducción de costos en el empaque.

Un factor cultural que incide en la decisión de compra de productos cosméticos es la percepción, actualmente el consumo de productos colombianos es más apetecido por países extranjeros ya que muchos de estos productos que se exportan se realizan a partir de bases naturales, por el contrario, en el mercado nacional, hoy por hoy la percepción es que el producto nacional es de mala calidad; así que en este orden de ideas los productos cosméticos de Colombia son apreciados más en el exterior que en el mercado local.

Dentro de las ventajas de las Mipymes del sector cosmético de Bogotá e incluso del país se encuentra en la gran diversidad natural que permite crear productos y fórmulas mejoradas con esencias o bases de productos naturales, la innovación de las empresas en cuanto a diseño y empaque, así como la creación de nuevas fórmulas acorde a las tendencias del mercado, el reconocimiento que ha tomado el país en calidad de producto cosmético, el apoyo de instituciones como la cámara de comercio y proexport (capacitaciones, asesorías) son las mayores ventajas con las que cuentan las empresas del clúster de cosmético de Bogotá; por el contrario dentro de las desventajas se encuentra el aumento de los impuestos a las empresas, las diligencias y requisitos para la exportación de productos a algunos países, la preferencia de los consumidores locales por producto cosmético de marcas extranjeras, la falta de capital para invertir en investigación y desarrollo de productos y las grandes cargas tributarias que afectan la producción, como ocurrió con el aumento del IVA del 16\% al 19\%.

\section{Referencias bibliográficas}

ANDI. (2018). ANDI. Obtenido de http://www.andi.com.co/Uploads/ANDI\%20Informe\%20 Coyuntura\%20Economica\%20-\%20Febrero\%202018.pdf

Arana , O., \& Ballesteros, A. (Junio de 2016). Los clúster como herramienta para dinamizar la competitividad. Dictamen Libre(18), 91. Recuperado el 2017, de file://C:/Users/pc/ Downloads/Dialnet-LosClusterComoHerramientaParaDinamizarLaCompetitiv-5710358.pdf 
Batres, R., \& Calderon, L. (2007). competitividad y desarrollo internacional. (E. Zuñiga, Ed.) Mexico D.f, Mexico: McGraw-Hill Interamericanaeditores S.A.

Cámara de Comercio de Bogotá. (s.f.). Cámara de Comercio de Bogotá. Recuperado el 2018, de https:// www.ccb.org.co/Clusters/Cluster-de-Cosmeticos/Sobre-el-Cluster/Quienes-somos

Camara de comercio de Bogotá. (03 de 2018). Camara de comercio de Bogotá. Recuperado el 2018, de https://www.ccb.org.co/Clusters/Cluster-de-Cosmeticos/Noticias/2018/Marzo-2018/Industria-cosmetica-y-de-aseo-retoma-rumbo-de-crecimiento

Canipec. (2017). CANIPEC. Recuperado el 2017, de http://canipec.org.mx/industria-mexicana-de-cuidado-personal-vende-bien-en-alianza-del-pacifico/

Capó, J., Expósito, M., \& Tomás, J. (2007). Creación de Redes Interorganizativas en un Cluster. Recuperado el 2017, de https://scielo.conicyt.cl/pdf/infotec/v18n5/art02.pdf

CCB. (2018). Camara de Comercio de Bogotá. Recuperado el 2018, de https://www.ccb.org.co/ Clusters/Cluster-de-Cosmeticos

CNN Español. (Junio de 2017). CNN Español. Recuperado el 2017, de https://cnnespanol.cnn. com/2017/06/30/el-precio-de-la-belleza-en-estos-paises-ser-bello-es-mas-barato/

Deming, E. (1989). Calidad, Productividad y Competitividad. (S. Diaz de Santos, Ed., \& J. Nicolau, Trad.) Ediciones Diaz de Santos, S.A.

Dinero. (28 de 09 de 2017). Dinero. Obtenido de https://www.dinero.com/edicion-impresa/negocios/ articulo/como-le-va-al-negocio-de-cosmeticos-y-aseo-en-colombia/250404

Expo Belleza Fest. (Febrero de 2018). Expansion. Recuperado el 2018, de https://expansion.mx/ economia/2018/02/23/mexico-se-pone-guapo-y-crece-11-al-ano-en-el-sector-cosmetico

Forbes Mexico. (Marzo de 2017). Forbes Mexico. Recuperado el 2017, de https://www.forbes.com. $\mathrm{mx} /$ forbes-life/belleza-lujo-necesario/

Innpulsa, Gobierno de Colombia, ClusterDevelopment, \& UniversidaddelRosario. (2018). Iniciativas Clúster en Colombia (Primera ed.). Bogota: Alma Digital s.a.s.

Jaramillo, A. (2017). Merca2.0. Recuperado el 2017, de https://www.merca20.com/cuales-son-loscosmeticos-que-mas-se-compran-en-mexico/

Krugman, P. (2007). comprender la globalizacion. Madrid, España: Alianza Editorial.

McCormick, D. (2005). El Futuro de los Clusters y las cadenas. Semestre Económico, 91. Obtenido de file:///C:/Users/pc/Downloads/Dialnet-EIFuturoDeLosClustersYLasCadenasProductivas-2929483.pdf 
Mincit. (2018). Mincit. Obtenido de http://www.mincit.gov.co/loader.php?IServicio=Documentos\&IFuncion=verPdf\&id=80988\&name=OEE_MA_JM_Estadisticas_de_comercio_exterior_ ene-may_2018.pdf\&prefijo=file

Nielsen. (Junio de 2016). Nielsen. Recuperado el 2017, de http://www.nielsen.com/co/es/insights/ news/2016/Marca-global-o-local-Que-prefieren-los-consumidores-colombianos.html

Nielsen. (2016). Nielsen. Recuperado el 2017, de http://www.nielsen.com/mx/es/insights/news/2016/ Mexicanos-prefieren-alimentos-frescos-de-marcas-y-empresas-locales.html

Portafolio. (25 de Marzo de 2018). Portafolio. Recuperado el 2018, de http://www.portafolio.co/ negocios/industria-cosmetica-y-de-aseo-retoma-rumbo-de-crecimiento-515554

Porter, M. (1990). La ventaja competitiva de las naciones. Buenos Aires, Argentina: Javier Vergara Editor Grupo Z.

Porter, M. (1998). La ventaja competitiva de las naciones. En M. Porter, \& Vergara (Ed.), La ventaja competitiva de las naciones (págs. 1-16). Buenos Aires, Argentina: Deusto.

Procolombia. (Marzo de 2018). Procolombia. Recuperado el 2018, de http://www.colombiatrade. com.co/noticias/cosmeticos-colombianos-una-oportunidad-de-negocio-en-el-exterior

Procolombia. (s.f). Procolombia. Recuperado el 2018, de http://www.procolombia.co/oportunidades-de-negocio-en-sector-cosmeticos-y-aseo-personal

Propais. (Diciembre de 2013). Oportunidades de negocio en sectoriales y grupos poblaciones clave. Recuperado el 2017, de http://propais.org.co/wp-content/uploads/inteligencia-mercados/ im2-oportunidades-sectoriales.pdf

RED CLUSTER Colombia. (s.f.). RED CLUSTER Colombia. Recuperado el 2018, de http://www. redclustercolombia.com/clusters-en-colombia/iniciativa/183

Region Central RAPE. (2016). Competitividad en la region central: balance y perfiles productivos de los territorios. Obtenido de https://campusvirtual.bancoldex.com/pluginfile.php/16087/mod_ resource/content/10/COMPETITIVIDAD\%20EN\%20LA\%20REGI\%C3\%93N\%20CENTRAL.pdf

Tamayo, M., \& Tamayo. (2002). La Investigacion Cientifica. En M. Tamayo, \& Tamayo, El proceso de la investigacion cientifica (cuarta ed., pág. 46). Limusa.

Taylor, S., \& Bogdan, R. (1992). LA ENTREVISTA EN PROFUNDIDAD . En S. Taylor, \& R. Bogdan, Introducción a los métodos cualitativos de investigación (págs. 100-132). España: Paidós. 